\title{
Supplemental Information: Concentrating Per- and Polyfluoroalkyl Substances (PFAS) in Municipal Solid Waste Landfill Leachate Using Foam Separation
}

Nicole M. Robey, Bianca F. da Silva, Michael D. Annable, Timothy G. Townsend, John A. Bowden* University of Florida Department of Environmental Engineering Sciences

Submitted to:

Environmental Science \& Technology

* Corresponding Author: john.bowden@ufl.edu

Total: 13 pages (1 Figure, 6 Tables)

Figure S1: Extraction efficiency experiment details

Table S1: Mass-labeled PFAS tracer compounds. The molecular formula includes deuterated/13C information from the manufacturer

Table S2: LC-MS/MS Analytical Conditions

Table S3: Target PFAS Analytes SRM experiment - Scan parameters

Table S4: PFAS standard compounds and corresponding mass-labeled compounds used to develop calibration curves.

Table S5: Extraction efficiency experiment result details.

Table S6: Comparison of landfill leachate extraction efficiency results from previous reports. 


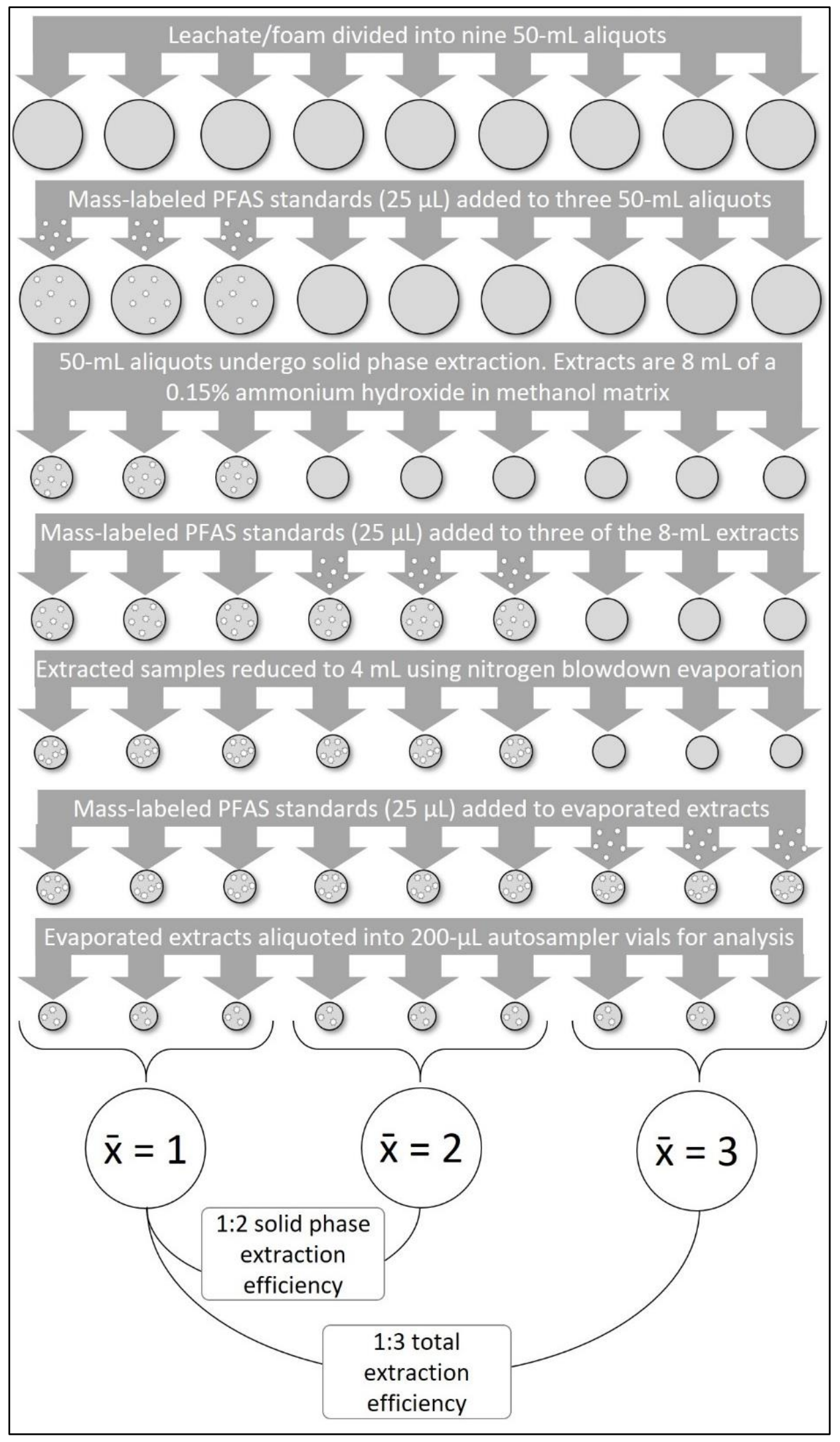

Figure S1: Extraction efficiency experiment details 
Table S1: Mass-labeled PFAS tracer compounds. The molecular formula includes deuterated $/{ }^{13} \mathrm{C}$ information from the manufacturer (Wellington Laboratories).

\begin{tabular}{|c|c|c|c|c|}
\hline Abbreviation & Compound & $\begin{array}{l}\text { Molecular } \\
\text { Formula }\end{array}$ & PFAS Type & $\begin{array}{l}\text { Concentration } \\
(\mathrm{ng} / \mathrm{g})\end{array}$ \\
\hline M4PFBA & perfluorobutanoic acid & ${ }^{13} \mathrm{C}_{4} \mathrm{HF}_{7} \mathrm{O}_{2}$ & carboxylate & 22.118 \\
\hline M5PFPeA & perfluoropentanoic acid & ${ }^{13} \mathrm{C}_{5} \mathrm{HF}_{9} \mathrm{O}_{3}$ & carboxylate & 22.118 \\
\hline M5PFHxA & perfluorohexanoic acid & ${ }^{13} \mathrm{C}_{5}{ }^{12} \mathrm{CHF}_{11} \mathrm{O}_{2}$ & carboxylate & 22.118 \\
\hline M4PFHpA & perfluoroheptanoic acid & ${ }^{13} \mathrm{C}_{4}{ }^{12} \mathrm{C}_{3} \mathrm{HF}_{13} \mathrm{O}_{2}$ & carboxylate & 22.118 \\
\hline M8PFOA & perfluorooctanoic acid & ${ }^{13} \mathrm{C}_{8} \mathrm{HF}_{15} \mathrm{O}_{2}$ & carboxylate & 22.118 \\
\hline M9PFNA & perfluorononanoic acid & ${ }^{13} \mathrm{C}_{9} \mathrm{HF}_{17} \mathrm{O}_{2}$ & carboxylate & 22.118 \\
\hline M6PFDA & perfluorodecanoic acid & ${ }^{13} \mathrm{C}_{6}{ }^{12} \mathrm{C}_{4} \mathrm{HF}_{19} \mathrm{O}_{2}$ & carboxylate & 22.118 \\
\hline M7PFUdA & perfluoroundecanoic acid & ${ }^{13} \mathrm{C}_{7}{ }^{12} \mathrm{C}_{4} \mathrm{HF}_{21} \mathrm{O}_{2}$ & carboxylate & 22.118 \\
\hline MPFDoA & perfluorododecanoic acid & ${ }^{13} \mathrm{C}_{2}{ }^{12} \mathrm{C}_{10} \mathrm{HF}_{23} \mathrm{O}_{2}$ & carboxylate & 22.118 \\
\hline M2PFTeDA & perfluorotetradecanoic acid & ${ }^{13} \mathrm{C}_{2}{ }^{12} \mathrm{C}_{12} \mathrm{HF}_{27} \mathrm{O}_{2}$ & carboxylate & 22.118 \\
\hline M8FOSA & perfluorooctane sulfonamide & ${ }^{13} \mathrm{C}_{8} \mathrm{H}_{2} \mathrm{~F}_{17} \mathrm{NO}_{2} \mathrm{~S}$ & sulfonamide & 22.118 \\
\hline d3-N-MeFOSAA & $\begin{array}{l}\text { n-methylperfluoro-1- } \\
\text { octanesulfonamidoacetic acid }\end{array}$ & $\mathrm{C}_{11} \mathrm{D}_{3} \mathrm{H}_{3} \mathrm{~F}_{17} \mathrm{NO}_{4} \mathrm{~S}$ & $\begin{array}{l}\text { sulfonamidoacetic } \\
\text { acid }\end{array}$ & 22.118 \\
\hline d5-N-EfFOSAA & $\begin{array}{l}\text { n-ethylperfluoro-1- } \\
\text { octanesulfonamidoacetic acid }\end{array}$ & $\mathrm{C}_{12} \mathrm{D}_{5} \mathrm{H}_{3} \mathrm{~F}_{17} \mathrm{NO}_{4} \mathrm{~S}$ & $\begin{array}{l}\text { sulfonamidoacetic } \\
\text { acid }\end{array}$ & 22.118 \\
\hline M3PFBS & perfluorobutyl sulfonate & ${ }^{13} \mathrm{C}_{3}{ }^{12} \mathrm{CHF}_{9} \mathrm{O}_{3} \mathrm{~S}$ & sulfonate & 20.547 \\
\hline M3PFHxS & perfluorohexyl sulfonate & ${ }^{13} \mathrm{C}_{3}{ }^{12} \mathrm{C}_{3} \mathrm{HF}_{13} \mathrm{O}_{3} \mathrm{~S}$ & sulfonate & 20.923 \\
\hline M8PFOS & perfluorooctyl sulfonate & ${ }^{13} \mathrm{C}_{8} \mathrm{HF}_{17} \mathrm{O}_{3} \mathrm{~S}$ & sulfonate & 21.167 \\
\hline M2-4:2FTS & 4:2 fluorotelomer sulfonate & ${ }^{13} \mathrm{C}_{2}{ }^{12} \mathrm{C}_{4} \mathrm{H}_{4} \mathrm{~F}_{9} \mathrm{O}_{3} \mathrm{SNa}$ & fluorotelomer & 20.680 \\
\hline M2-6:2FTS & 6:2 fluorotelomer sulfonate & ${ }^{13} \mathrm{C}_{2}{ }^{12} \mathrm{C}_{6} \mathrm{H}_{4} \mathrm{~F}_{13} \mathrm{O}_{3} \mathrm{SNa}$ & fluorotelomer & 20.990 \\
\hline M2-8:2FTS & $8: 2$ fluorotelomer sulfonate & ${ }^{13} \mathrm{C}_{2}{ }^{12} \mathrm{C}_{8} \mathrm{H}_{4} \mathrm{~F}_{17} \mathrm{O}_{3} \mathrm{SNa}$ & fluorotelomer & 21.189 \\
\hline MFHEA & $\begin{array}{l}\text { 6:2 fluorotelomer carboxylic } \\
\text { acid }\end{array}$ & ${ }^{13} \mathrm{C}_{2}{ }^{12} \mathrm{C}_{6} \mathrm{H}_{3} \mathrm{~F}_{13} \mathrm{O}_{2}$ & telomer acid & 23.002 \\
\hline MFOEA & n-2-perfluorooctyl ethanoic acid & ${ }^{13} \mathrm{C}_{2}{ }^{12} \mathrm{C}_{8} \mathrm{H}_{3} \mathrm{~F}_{17} \mathrm{O}_{2}$ & telomer acid & 23.002 \\
\hline MFDEA & n-2-perfluorodecyl ethanoic acid & ${ }^{13} \mathrm{C}_{2}{ }^{12} \mathrm{C}_{10} \mathrm{H}_{3} \mathrm{~F}_{21} \mathrm{O}_{2}$ & $\begin{array}{l}\text { sulfonamidoacetic } \\
\text { acid }\end{array}$ & 23.002 \\
\hline M3HFPO-DA & $\begin{array}{l}\text { hexafluoropropylene oxide } \\
\text { dimer acid (Gen-X) }\end{array}$ & ${ }^{13} \mathrm{C}_{3}{ }^{12} \mathrm{C}_{3} \mathrm{HF}_{11} \mathrm{O}_{3}$ & $\mathrm{n} / \mathrm{a}$ & 111.026 \\
\hline $\begin{array}{l}\text { d3-N-MeFOSA- } \\
\text { M }\end{array}$ & $\begin{array}{l}\text { n-methylperfluorooctane-1- } \\
\text { sulfonamide }\end{array}$ & $\mathrm{C}_{9} \mathrm{D}_{3} \mathrm{HF}_{17} \mathrm{NO}_{2} \mathrm{~S}$ & sulfonamide & 118.144 \\
\hline d5-N-EtFOSA-M & $\begin{array}{l}\text { n-ethylperfluorooctane-1- } \\
\text { sulfonamide }\end{array}$ & $\mathrm{C}_{10} \mathrm{D}_{5} \mathrm{HF}_{17} \mathrm{NO}_{2} \mathrm{~S}$ & sulfonamide & 111.379 \\
\hline
\end{tabular}


Table S2: LC-MS/MS Analytical Conditions

\begin{tabular}{|c|c|}
\hline \multicolumn{2}{|r|}{ Chromatography Conditions - Thermo Scientific Vanquish UHPLC } \\
\hline \multirow{5}{*}{ LC conditions } & 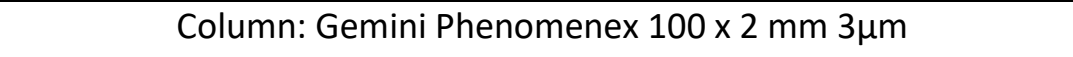 \\
\hline & $\begin{array}{l}\text { Gradient elution using Water and methanol both containing } 5 \mathrm{mM} \text { of } \\
\text { ammonium acetate }\end{array}$ \\
\hline & Oven temperature $40^{\circ} \mathrm{C}$ \\
\hline & Flow rate: $0.5 \mathrm{mLmin}^{-1}$ \\
\hline & Injection volume: $10 \mu \mathrm{L}$ \\
\hline \multicolumn{2}{|r|}{ Mass Spectrometry Analysis - Thermo Quantis } \\
\hline \multirow{6}{*}{$\begin{array}{l}\text { Ion Source } \\
\text { parameters: }\end{array}$} & Electrospray - negative mode \\
\hline & Ion Spray : $1500 \mathrm{~V}$ \\
\hline & Sheath Gas: 50 Arb \\
\hline & Auxilliar Gas: 10 Arb \\
\hline & Ion Transfer tube Temperature: $250{ }^{\circ} \mathrm{C}$ \\
\hline & Vaporizer temperature: $550{ }^{\circ} \mathrm{C}$ \\
\hline
\end{tabular}


Table S3: Target PFAS Analytes SRM experiment - Scan parameters

\begin{tabular}{|c|c|c|c|c|}
\hline $\begin{array}{c}\text { Analytes } \\
\text { (abbreviation) }\end{array}$ & $\begin{array}{l}\text { Precursor } \\
\text { Ion }(m / z)\end{array}$ & $\begin{array}{l}\text { Fragment lon } \\
\qquad(\mathrm{m} / \mathrm{z})\end{array}$ & $\begin{array}{l}\text { Collision } \\
\text { Energy (V) }\end{array}$ & RF Lens (V) \\
\hline PFBA & 213 & 168.9 & 11 & 75 \\
\hline M3PFBA & 216 & 172 & 11 & 75 \\
\hline M4PFBA & 217 & 172 & 11 & 75 \\
\hline L-PFPrS-1 & 249.05 & 79.9 & 28.84 & 138 \\
\hline L-PFPrS-2 & 249.05 & 98.9 & 25.68 & 138 \\
\hline PFPeA & 263 & 219 & 11 & 64 \\
\hline M5PFPeA & 268 & 223 & 11 & 64 \\
\hline FBSA-1 & 297.95 & 77.9 & 24.8 & 144 \\
\hline FBSA-2 & 297.95 & 118.9 & 17.97 & 144 \\
\hline L-PFBS-1 & 298.975 & 79.9 & 32 & 152 \\
\hline L-PFBS-2 & 298.975 & 98.9 & 28.46 & 152 \\
\hline M3PFBS & 302 & 99 & 29 & 152 \\
\hline PFHxA & 312.9 & 269 & 10 & 73 \\
\hline M5PFHxA & 318 & 273 & 10 & 73 \\
\hline $4: 2 F T S-2$ & 326.962 & 81 & 28.5 & 138 \\
\hline $4: 2 \mathrm{FTS}-1$ & 326.962 & 306.8 & 17.97 & 138 \\
\hline M2-4:2FTS & 329 & 81 & 17.97 & 138 \\
\hline HFPO-DA & 329 & 285 & 7 & 65 \\
\hline PFPeS-1 & 348.975 & 79.9 & 34.28 & 174 \\
\hline PFPeS-2 & 348.975 & 98.9 & 31.45 & 174 \\
\hline PFHpA-1 & 362.9 & 169 & 17 & 81 \\
\hline PFHpA-2 & 362.9 & 318.9 & 11 & 81 \\
\hline M4PFHpA & 367 & 322 & 11 & 81 \\
\hline FHEA & 376.9 & 293 & 20 & 100 \\
\hline NaDONA & 377 & 251 & 10 & 280 \\
\hline
\end{tabular}




\begin{tabular}{|c|c|c|c|c|}
\hline $\begin{array}{c}\text { Analytes } \\
\text { (abbreviation) }\end{array}$ & $\begin{array}{l}\text { Precursor } \\
\text { Ion }(m / z)\end{array}$ & $\begin{array}{l}\text { Fragment lon } \\
\qquad(\mathrm{m} / \mathrm{z})\end{array}$ & $\begin{array}{l}\text { Collision } \\
\text { Energy (V) }\end{array}$ & RF Lens (V) \\
\hline FHxSA-1 & 397.96 & 77.9 & 28.42 & 185 \\
\hline FHxSA-2 & 397.96 & 377.9 & 20.33 & 185 \\
\hline L-PFHxS-2 & 398.975 & 79.9 & 37.48 & 174 \\
\hline L-PFHxS-1 & 398.975 & 98.9 & 34.95 & 174 \\
\hline M3PFHxS & 402 & 99 & 35 & 151 \\
\hline PFOA-1 & 413 & 169.0 & 19 & 94 \\
\hline PFOA-2 & 413 & 368.9 & 11 & 94 \\
\hline M2PFOA & 415 & 370 & 11 & 94 \\
\hline M8PFOA & 421 & 376 & 11 & 94 \\
\hline $6: 2 \mathrm{FTS}-2$ & 426.962 & 80.9 & 29.94 & 166 \\
\hline $6: 2 \mathrm{FTS}-1$ & 426.962 & 406.9 & 21.39 & 166 \\
\hline M2-6:2FTS-2 & 429 & 81 & 21.39 & 166 \\
\hline M2-6:2FTS-1 & 429 & 376 & 21.39 & 166 \\
\hline PFHpS-1 & 448.925 & 79.9 & 39.2 & 223 \\
\hline PFHpS-2 & 448.925 & 98.9 & 37.56 & 223 \\
\hline FOUEA & 456.9 & 392.9 & 12 & 100 \\
\hline PFECHS-2 & 460.925 & 98.9 & 28.76 & 171 \\
\hline PFECHS-1 & 460.925 & 380.9 & 26.15 & 171 \\
\hline PFNA-1 & 463 & 218.9 & 16 & 101 \\
\hline PFNA-2 & 463 & 418.9 & 12 & 101 \\
\hline M9PFNA & 472 & 427 & 12 & 101 \\
\hline FOEA & 476.9 & 393 & 18 & 100 \\
\hline MFOEA-1 & 479.01 & 394 & 11 & 107 \\
\hline N-AP-FHxSA-1 & 483.05 & 168.9 & 26.57 & 244 \\
\hline N-AP-FHxSA-2 & 483.05 & 318.9 & 21.55 & 244 \\
\hline FOSA-I-1 & 497.962 & 77.9 & 30.44 & 206 \\
\hline
\end{tabular}




\begin{tabular}{|c|c|c|c|c|}
\hline $\begin{array}{c}\text { Analytes } \\
\text { (abbreviation) }\end{array}$ & $\begin{array}{l}\text { Precursor } \\
\text { Ion }(m / z)\end{array}$ & $\begin{array}{l}\text { Fragment Ion } \\
\qquad(m / z)\end{array}$ & $\begin{array}{l}\text { Collision } \\
\text { Energy (V) }\end{array}$ & RF Lens (V) \\
\hline FOSA-I-2 & 497.962 & 477.9 & 23.16 & 206 \\
\hline L-PFOS-1 & 498.925 & 79.9 & 40.26 & 214 \\
\hline L-PFOS-2 & 498.925 & 98.9 & 40.55 & 214 \\
\hline MPFOS-2 & 502.925 & 79.9 & 41.86 & 266 \\
\hline MPFOS-1 & 502.925 & 98.8 & 41.86 & 266 \\
\hline M8FOSA-I-2 & 505.95 & 77.9 & 30.4 & 196 \\
\hline M8FOSA-I-1 & 505.95 & 485.9 & 22.94 & 196 \\
\hline M8PFOS-1 & 507 & 80 & 44 & 259 \\
\hline M8PFOS-2 & 507 & 99 & 44 & 259 \\
\hline N-MeFOSA-M-2 & 511.962 & 168.9 & 26.23 & 187 \\
\hline N-MeFOSA-M-1 & 511.962 & 219 & 23.62 & 187 \\
\hline PFDA & 513 & 469 & 11 & 94 \\
\hline $8 \mathrm{Cl}-\mathrm{PFOS}-1$ & 514.925 & 79.9 & 41.44 & 273 \\
\hline $8 \mathrm{Cl}-\mathrm{PFOS}-2$ & 514.925 & 98.9 & 40.3 & 273 \\
\hline $\begin{array}{c}\text { d-N-MeFOSA-M- } \\
2\end{array}$ & 514.995 & 168.9 & 26.23 & 194 \\
\hline $\begin{array}{c}\text { d-N-MeFOSA-M- } \\
1\end{array}$ & 514.995 & 218.9 & 23.74 & 194 \\
\hline MPFDA & 515 & 470 & 11 & 94 \\
\hline M6PFDA & 519 & 474 & 11 & 94 \\
\hline N-EtFOSA-M-2 & 525.962 & 168.9 & 26.69 & 209 \\
\hline N-EtFOSA-M-1 & 525.962 & 219 & 24.04 & 209 \\
\hline $8: 2 F T S-2$ & 526.988 & 486.8 & 30.57 & 179 \\
\hline $8: 2 \mathrm{FTS}-1$ & 526.988 & 506.8 & 24.76 & 179 \\
\hline M2-8:2FTS & 529 & 81 & 24.76 & 179 \\
\hline 9Cl-PF3ONS-1 & 530.838 & 350.9 & 24.71 & 155 \\
\hline
\end{tabular}




\begin{tabular}{|c|c|c|c|c|}
\hline $\begin{array}{c}\text { Analytes } \\
\text { (abbreviation) }\end{array}$ & $\begin{array}{l}\text { Precursor } \\
\text { Ion }(m / z)\end{array}$ & $\begin{array}{l}\text { Fragment Ion } \\
\qquad(m / z)\end{array}$ & $\begin{array}{l}\text { Collision } \\
\text { Energy (V) }\end{array}$ & RF Lens (V) \\
\hline d-N-EtFOSA-M-3 & 530.925 & 169 & 26.69 & 203 \\
\hline d-N-EtFOSA-M-2 & 530.925 & 219 & 24.76 & 203 \\
\hline L-PFNS-1 & 548.975 & 80 & 42.99 & 280 \\
\hline L-PFNS-2 & 548.975 & 98.9 & 42.78 & 280 \\
\hline FOSAA-2 & 555.95 & 418.9 & 23.45 & 210 \\
\hline FOSAA-1 & 555.95 & 497.8 & 26.06 & 210 \\
\hline FDUEA & 557 & 493 & 21 & 280 \\
\hline PFUnDA & 563 & 518.9 & 11 & 116 \\
\hline N-MeFOSAA-1 & 569.975 & 418.8 & 18.18 & 178 \\
\hline N-MeFOSAA-2 & 569.975 & 482.8 & 14.39 & 178 \\
\hline M7PFUnDA-2 & 570 & 525 & 11 & 116 \\
\hline $\begin{array}{c}\text { d3-N-MeFOSAA- } \\
1\end{array}$ & 572.962 & 418.9 & 18.56 & 174 \\
\hline $\begin{array}{c}\text { d3-N-MeFOSAA- } \\
2\end{array}$ & 572.962 & 482.8 & 14.06 & 174 \\
\hline FDEA & 576.8 & 493 & 8 & 100 \\
\hline N-EtFOSAA-2 & 583.962 & 418.9 & 18.52 & 179 \\
\hline N-EtFOSAA-1 & 583.962 & 525.9 & 18.1 & 179 \\
\hline d5-N-EtFOSAA-2 & 589.012 & 418.9 & 19.15 & 176 \\
\hline d5-N-EtFOSAA-1 & 589.012 & 530.9 & 19.36 & 176 \\
\hline L-PFDS-1 & 598.925 & 79.9 & 44.51 & 280 \\
\hline L-PFDS-2 & 598.925 & 98.9 & 46.87 & 280 \\
\hline PFDoDA-2 & 612.925 & 318.9 & 17.51 & 129 \\
\hline PFDoDA-1 & 612.925 & 568.9 & 9.8 & 129 \\
\hline MPFDoA & 615 & 570 & 9.8 & 129 \\
\hline 10:2FTS-2 & 626.95 & 80.9 & 34.19 & 280 \\
\hline
\end{tabular}




\begin{tabular}{|c|c|c|c|c|}
\hline $\begin{array}{c}\text { Analytes } \\
\text { (abbreviation) }\end{array}$ & $\begin{array}{l}\text { Precursor } \\
\text { Ion }(m / z)\end{array}$ & $\begin{array}{l}\text { Fragment lon } \\
\qquad(\mathrm{m} / \mathrm{z})\end{array}$ & $\begin{array}{l}\text { Collision } \\
\text { Energy (V) }\end{array}$ & RF Lens (V) \\
\hline 10:2FTS-1 & 626.95 & 606.8 & 29.01 & 280 \\
\hline 11Cl-PF3OUdS & 630.95 & 450.8 & 27.2 & 225 \\
\hline SAmPAP-1 & 650 & 526 & 25 & 280 \\
\hline PFTrDA-2 & 662.812 & 318.9 & 18.98 & 136 \\
\hline PFTrDA-1 & 662.812 & 618.9 & 10.73 & 136 \\
\hline L-PFDoS-1 & 698.925 & 80 & 55 & 280 \\
\hline L-PFDoS-3 & 698.925 & 99 & 55 & 280 \\
\hline 6:6PFPi & 700.9 & 400.9 & 55 & 188 \\
\hline PFTeDA & 713 & 669 & 12 & 107 \\
\hline M2PFTeDA & 715 & 670 & 12 & 107 \\
\hline 6:2diPAP-2 & 788.925 & 96.9 & 29.3 & 216 \\
\hline 6:2diPAP-1 & 788.925 & 442.9 & 17.05 & 216 \\
\hline 6:8PFPi-2 & 800.9 & 400.9 & 55 & 188 \\
\hline 6:8PFPi-1 & 800.9 & 500.8 & 55 & 188 \\
\hline PFHxDA-2 & 812.875 & 418.9 & 20.42 & 167 \\
\hline PFHxDA-1 & 812.875 & 768.8 & 11.95 & 167 \\
\hline $6: 2 / 8: 2$ diPAP & 889 & 443 & 20 & 280 \\
\hline PFODA-2 & 912.888 & 318.8 & 25.05 & 188 \\
\hline PFODA-1 & 912.888 & 868.8 & 13.3 & 188 \\
\hline $8: 2$ diPAP-2 & 988.888 & 522.9 & 27.11 & 280 \\
\hline 8:2diPAP & 989 & 543 & 20 & 280 \\
\hline diSAmPAP & 1202.95 & 525.9 & 25 & 280 \\
\hline
\end{tabular}

*-1 and -2 means first and second transition of the analyte - first transition used for quantitation and second transition for confirmation. 
Table S4: PFAS standard compounds and corresponding mass-labeled compounds used to develop calibration curves. When the same compound was not available as mass-labeled, the closest retention time labeled compound was selected. Detection and quantification limits (LOD and LOQ) reflect concentrations in the 4- $\mathrm{mL}$ solvent-eluted samples. In order to compare these values to leachate concentrations, multiply the instrument detection limit by the ratio between volumes, in this case, $4 \mathrm{~mL}$ divided by the extracted volume of each leachate sample (approximately $50 \mathrm{~mL}$ ).

\begin{tabular}{|c|c|c|c|c|c|}
\hline Abbreviation & Compound & PFAS Type & $\begin{array}{l}\text { Correlative } \\
\text { Labeled } \\
\text { Compound }\end{array}$ & LOD (ng/L) & $\begin{array}{l}\mathrm{LOQ} \\
\text { (ng/L) }\end{array}$ \\
\hline PFBA & perfluorobutanoic acid & carboxylate & M4PFBA & 4.18 & 8.09 \\
\hline PFPrS & perfluoropropane sulfonate & sulfonate & M5PFPeA & 1.66 & 6.42 \\
\hline PFPeA & perfluoropentanoic acid & carboxylate & M5PFPeA & 20.92 & 182.15 \\
\hline PFBS & perfluorobutyl sulfonate & sulfonate & M3PFBS & 3.70 & 18.52 \\
\hline FBSA & perfluorobutane sulfonamide & sulfonamide & M3PFBS & 80.74 & 398.28 \\
\hline $4: 2 \mathrm{FTS}$ & 4:2 fluorotelomer sulfonate & telomer sulfonate & M24:2FTS & 3.91 & 19.56 \\
\hline PFHxA & perfluorohexanoic acid & carboxylate & M5PFHxA & 4.18 & 20.92 \\
\hline PFPeS & perfluoropentane sulfonate & sulfonate & M3HFPO-DA & 3.93 & 19.67 \\
\hline PFHXS & perfluorohexyl sulfonate & sulfonate & M3PFHxS & 19.08 & 35.46 \\
\hline PFHpA & perfluoroheptanoic acid & carboxylate & M4PFHpA & 4.18 & 20.92 \\
\hline NaDONA & $\begin{array}{l}\text { dodecafluoro-3H-4,8- } \\
\text { dioxanonanoate } \\
\end{array}$ & other & M4PFHpA & 76.85 & 379.09 \\
\hline FHEA & 6:2 fluorotelomer carboxylic acid & telomer acid & M4PFHpA & 35.40 & 165.86 \\
\hline AP-FHXSA & & & M26:2FTS & 32.48 & 362.66 \\
\hline PFECHS & $\begin{array}{l}\text { n-decafluoro-4 } \\
\text { ethylcyclohexanesulfonate }\end{array}$ & cyclic & M26:2FTS & 3.32 & 16.59 \\
\hline FHxSA & perfluorohexane sulfonamide & sulfonamide & M3PFHXS & 9.76 & 36.26 \\
\hline $6: 2 \mathrm{FTS}$ & 6:2 fluorotelomer sulfonate & telomer sulfonate & M26:2FTS & 3.97 & 19.87 \\
\hline PFHpS & perfluoroheptane sulfonate & sulfonate & M26:2FTS & 9.94 & 36.93 \\
\hline PFOA & perfluorooctanoic acid & carboxylate & M8PFOA & 10.46 & 38.88 \\
\hline PFOS & perfluorooctyl sulfonate & sulfonate & M8PFOS & 19.36 & 81.44 \\
\hline PFNA & perfluorononanoic acid & carboxylate & M9PFNA & 4.18 & 20.92 \\
\hline Cl-PFOS & $\begin{array}{l}\text { 8-chlorohexadecafluoro-3- } \\
\text { oxaoctane-1-sulfonate }\end{array}$ & other & M8PFOS & 17.06 & 71.76 \\
\hline FOEA & n-2-perfluorooctyl ethanoic acid & telomer acid & MFOEA & 35.92 & 168.30 \\
\hline FOUEA & 2H-perfluoro-2-decanoic acid & $\begin{array}{l}\text { unsaturated telomer } \\
\text { acid }\end{array}$ & MFOEA & 3.18 & 6.14 \\
\hline Cl-PF3ONS & $\begin{array}{l}\text { 9-chlorohexadecafluoro-3- } \\
\text { oxanonane-1-sulfonate }\end{array}$ & other & M28:2FTS & 3.30 & 6.38 \\
\hline FOSAA & $\begin{array}{l}\text { 2-perfluorooctanesulfonamido } \\
\text { acetic acid }\end{array}$ & sulfonamidoacetic acid & M28:2FTS & 8.83 & 17.65 \\
\hline PFNS & perfluorononane sulfonate & sulfonate & M28:2FTS & 10.04 & 84.48 \\
\hline $8: 2 \mathrm{FTS}$ & 8:2 fluorotelomer sulfonate & telomer sulfonate & M28:2FTS & 20.08 & 37.32 \\
\hline FOSA & perfluoro-1-octanesulfonamide & sulfonamide & M8FOSA & 8.09 & 38.88 \\
\hline PFDA & Perfluorodecanoic acid & carboxylate & M6PFDA & 1.62 & 8.09 \\
\hline N-MeFOSAA & $\begin{array}{l}\text { N-methylperfluoro-1- } \\
\text { octanesulfonamidoacetic acid }\end{array}$ & sulfonamidoacetic acid & $\begin{array}{l}\text { d3-N- } \\
\text { MeFOSAA }\end{array}$ & 8.09 & 88.00 \\
\hline
\end{tabular}




\begin{tabular}{|c|c|c|c|c|c|}
\hline Abbreviation & Compound & PFAS Type & $\begin{array}{l}\text { Correlative } \\
\text { Labeled } \\
\text { Compound }\end{array}$ & LOD (ng/L) & $\begin{array}{l}\text { LOQ } \\
\text { (ng/L) }\end{array}$ \\
\hline PFDS & perfluorodecane sulfonate & sulfonate & $\begin{array}{l}\text { d5-N- } \\
\text { EtFOSAA }\end{array}$ & 20.19 & 84.92 \\
\hline PFUnDA & perfluoroundecanoic acid & carboxylate & M7PFUnDA & 4.18 & 20.92 \\
\hline N-EtFOSAA & $\begin{array}{l}\text { N-ethylperfluoro-1- } \\
\text { octanesulfonamidoacetic acid }\end{array}$ & sulfonamidoacetic acid & $\begin{array}{l}\text { d5-N- } \\
\text { EtFOSAA }\end{array}$ & 20.92 & 88.00 \\
\hline FDUEA & n-2-perfluorodecyl ethanoic acid & $\begin{array}{l}\text { unsaturated telomer } \\
\text { acid }\end{array}$ & M7PFUnDA & 1711.28 & $\begin{array}{l}13092.9 \\
4\end{array}$ \\
\hline FDEA & n-2-perfluorodecyl ethanoic acid & saturated telomer acid & M7PFUnDA & 35.67 & 167.14 \\
\hline Cl-PF3OUdS & $\begin{array}{l}\text { 11-chloroeicosafluoro-3- } \\
\text { oxaundecane-1-sulfonate }\end{array}$ & other & $\begin{array}{l}\mathrm{d} 5-\mathrm{N}- \\
\text { EtFOSAA }\end{array}$ & 3.39 & 6.55 \\
\hline N-MeFOSA & $\begin{array}{l}\text { N-methylperfluorooctane } \\
\text { sulfonamide }\end{array}$ & sulfonamide & d-N-MeFOSA & 3.58 & 33.29 \\
\hline PFDoDA & perfluorododecanoic acid & carboxylate & MPFDoDA & 4.18 & 8.09 \\
\hline $10: 2 \mathrm{FTS}$ & 10:2 fluorotelomer sulfonate & telomer sulfonate & MPFDoDA & 8.68 & 32.24 \\
\hline 6:6PFPi & $\begin{array}{l}\text { sodium } \\
\text { bis(perfluorohexyl)phosphinate }\end{array}$ & $\begin{array}{l}\text { perfluoroalkylphosphin } \\
\text { ate }\end{array}$ & MPFDoDA & 3.45 & 17.23 \\
\hline N-EtFOSA & $\begin{array}{l}\text { N-ethylperfluorooctane } \\
\text { sulfonamide }\end{array}$ & sulfonamide & d-N-EtFOSA & 17.43 & 32.39 \\
\hline PFDoDS & perfluorododecane sulfonate & sulfonate & MPFDoDA & 17.13 & 31.83 \\
\hline PFTriDA & perfluorotridecanoic acid & carboxylate & d-N-EtFOSA & 6.97 & 8.09 \\
\hline 6:2diPAP & $\begin{array}{l}\text { 6:2 fluorotelomer phosphate } \\
\text { diester }\end{array}$ & phosphate ester & M2PFTeDA & 3.66 & 18.29 \\
\hline PFTreDA & perfluorotetradecanoic acid & carboxylate & M2PFTeDA & 4.18 & 20.92 \\
\hline 6:8PFPi & $\begin{array}{l}\text { sodium } \\
\text { perfluorohexylperfluorooctylphos } \\
\text { phinate }\end{array}$ & $\begin{array}{l}\text { perfluoroalkylphosphin } \\
\text { ate }\end{array}$ & M2PFTeDA & 1.78 & 8.89 \\
\hline 6:2/8:2diPAP & & & M2PFTeDA & 6.94 & 33.34 \\
\hline PFHXDA & perfluorohexadecanoic acid & carboxylate & M2PFTeDA & 3.16 & 6.12 \\
\hline 8:2diPAP & $\begin{array}{l}\text { 6:2 fluorotelomer phosphate } \\
\text { diester }\end{array}$ & phosphate ester & M2PFTeDA & 9.00 & 33.46 \\
\hline PFODA & perfluorooctadecanoic acid & carboxylate & M2PFTeDA & 3.51 & 6.80 \\
\hline diSAmPAP & $\begin{array}{l}\text { sodium bis-(2-N- } \\
\text { ethylperfluorooctane-1- } \\
\text { sulfonamido)ethyl }\end{array}$ & phosphate ester & M2PFTeDA & 15.68 & 29.13 \\
\hline
\end{tabular}


Table S5: Extraction efficiency experiment result details. Compounds are listed in increasing order of retention time.

\begin{tabular}{|c|c|c|c|c|c|c|c|c|}
\hline \multirow[b]{2}{*}{ Compound } & \multicolumn{3}{|c|}{ Extraction Efficiency (\%) } & \multicolumn{3}{|c|}{ Total Extraction Efficiency (\%) } & \multicolumn{2}{|c|}{ Evaporation Loss (\%) } \\
\hline & Leachate & Foam & $\Delta$ & Leachate & Foam & $\Delta$ & Leachate & Foam \\
\hline PFBA & 114 & 147 & 34 & 107 & 95 & -12 & 6 & 52 \\
\hline PFPeA & 104 & 115 & 11 & 88 & 84 & -4 & 16 & 31 \\
\hline PFBS & 84 & 131 & 47 & 73 & 100 & 27 & 11 & 31 \\
\hline PFHxA & 93 & 106 & 14 & 79 & 91 & 11 & 14 & 16 \\
\hline PFHxS & 106 & 128 & 21 & 80 & 92 & 12 & 26 & 36 \\
\hline PFHpA & 102 & 113 & 11 & 82 & 93 & 11 & 21 & 20 \\
\hline $6: 2$ FTS & 84 & 99 & 16 & 70 & 85 & 15 & 13 & 14 \\
\hline PFOA & 101 & 108 & 6 & 80 & 88 & 7 & 21 & 20 \\
\hline PFOS & 104 & 108 & 5 & 73 & 104 & 31 & 31 & 4 \\
\hline PFNA & 100 & 110 & 10 & 77 & 88 & 11 & 22 & 22 \\
\hline FOEA & 85 & 90 & 5 & 71 & 73 & 2 & 14 & 16 \\
\hline $8: 2$ FTS & 75 & 93 & 18 & 51 & 86 & 35 & 24 & 7 \\
\hline FOSA & 64 & 59 & -4 & 46 & 46 & 0 & 17 & 13 \\
\hline PFDA & 85 & 84 & -1 & 61 & 69 & 8 & 24 & 14 \\
\hline N-MeFOSAA & 73 & 79 & 6 & 55 & 64 & 10 & 18 & 15 \\
\hline N-Et-FOSAA & 72 & 66 & -7 & 53 & 54 & 1 & 20 & 12 \\
\hline PFUnDA & 65 & 64 & -1 & 45 & 51 & 6 & 20 & 13 \\
\hline N-MeFOSA & 57 & 54 & -3 & 38 & 42 & 5 & 20 & 12 \\
\hline PFDoDA & 44 & 43 & -1 & 31 & 34 & 3 & 13 & 9 \\
\hline N-EtFOSA & 54 & 42 & -12 & 37 & 35 & -2 & 16 & 7 \\
\hline PFTeDA & 19 & 17 & -2 & 14 & 14 & 0 & 6 & 3 \\
\hline MEAN & 80.1 & 88.3 & 8.2 & 62.4 & 70.8 & 8.4 & 17.7 & 17.5 \\
\hline
\end{tabular}


Table S6: Comparison of landfill leachate extraction efficiency results from previous reports, values are in \%. The comparison was made for PFAS evaluated in common with this study, most of these studies included additional compounds not listed here. Current solid phase extraction methods for PFAS in leachate vary between researchers. The procedure used in this study features commonly employed materials, such as WAX cartridges ${ }^{1,2}$, methanolic ammonium hydroxide ${ }^{1,2}$ (however, the concentration varies), and methanol, which was used in some portion of all SPE approaches referenced. Other efficiency experiment results in the literature, which were not included in this table, such as Li et al. ${ }^{5}$, which reported a range of recoveries (20 $\%$ to $120 \%$ ) for all compounds and not individual PFAS, and Gonzalez- Barreiro et al. ${ }^{6}$, which also reported similar recoveries for two SPE methods (ranging from $11 \%$ to $96 \%$ for Method A and $8 \%$ to $102 \%$ for Method B) using wastewater effluent, had comparable results. Optimization of PFAS extraction protocols for multiple compounds with a wide variety of extraction behaviors is a challenge, and thus, solid phase extraction efficiency for different compounds varies widely (i.e., from $8 \%$ to $160 \%$ ) among these studies; this method resulted in a comparable range (19\% to $114 \%$ ). Community-wide efforts aimed at harmonizing/standardizing PFAS measurement in leachate (e.g., development of a Standard Reference Material) would provide a means to better evaluate these workflows.

\begin{tabular}{|l|l|l|l|l|l|}
\hline & This study & $\begin{array}{c}\text { Benskin et al. } \\
(2012)^{1}\end{array}$ & \multicolumn{1}{|c|}{$\begin{array}{c}\text { Busch et al. } \\
(2010)^{2}\end{array}$} & Huset et al. (2011) & \multicolumn{1}{|c|}{$\begin{array}{c}\text { Allred et al. } \\
(2014)^{4}\end{array}$} \\
\hline Extraction Type & SPE & SPE & SPE & SPE & LLE \\
\hline PFBA & 114 & 54 & $53 \pm 21$ & $25 \pm 5$ & 11 \\
\hline PFPeA & 104 & $68^{*}$ & & $39 \pm 8$ & 41 \\
\hline PFBS & 84 & $68^{*}$ & & $54 \pm 2$ & \\
\hline PFHxA & 93 & 121 & $47 \pm 17$ & $64 \pm 9$ & 92 \\
\hline PFHxS & 106 & $102^{*}$ & $49 \pm 19$ & $80 \pm 3$ & 100 \\
\hline PFHpA & 102 & $137^{*}$ & & $110 \pm 12$ & \\
\hline $6: 2$ FTS & 84 & & & $74 \pm 2$ & 100 \\
\hline PFOA & 101 & 136 & $50 \pm 19$ & $72 \pm 16$ & 100 \\
\hline PFOS & 104 & 96 & $60 \pm 24$ & $69 \pm 1$ & 110 \\
\hline PFNA & 100 & 139 & $54 \pm 23$ & $120 \pm 18$ & 100 \\
\hline FOEA & 85 & 103 & $45 \pm 22$ & & 110 \\
\hline $8: 2$ FTS & 75 & & & $94 \pm 7$ & \\
\hline FOSA & 64 & $132^{*}$ & & $110 \pm 8$ & 110 \\
\hline PFDA & 85 & $146^{*}$ & $48 \pm 22$ & $73 \pm 7$ & 100 \\
\hline MeFOSAA & 73 & $123^{*}$ & & $71 \pm 4$ & 110 \\
\hline EtFOSAA & 72 & $116^{*}$ & & $65 \pm 3$ & 100 \\
\hline PFUnDA & 65 & $160^{*}$ & $47 \pm 20$ & $44 \pm 3$ & 100 \\
\hline MeFOSA & 57 & & $60 \pm 23$ & & \\
\hline PFDoA & 44 & $144^{*}$ & $45 \pm 20$ & $34 \pm 6$ & \\
\hline EtFOSA & 54 & & $54 \pm 18$ & & \\
\hline PFTeDA & 19 & $155^{*}$ & & $8 \pm 3$ & \\
\hline
\end{tabular}

* Results for these compounds are for non-mass-labeled standards, the remaining extraction efficiencies are for mass-labeled. 
References

(1) Benskin, J. P.; Ikonomou, M. G.; Woudneh, M. B.; Cosgrove, J. R. Rapid Characterization of Perfluoralkyl Carboxylate, Sulfonate, and Sulfonamide Isomers by High-Performance Liquid Chromatography-Tandem Mass Spectrometry. J. Chromatogr. A 2012, 1247, 165-170. https://doi.org/10.1016/j.chroma.2012.05.077.

(2) Busch, J.; Ahrens, L.; Sturm, R.; Ebinghaus, R. Polyfluoroalkyl Compounds in Landfill Leachates. Environ. Pollut. 2010, 158 (5), 1467-1471. https://doi.org/10.1016/j.envpol.2009.12.031.

(3) Huset, C. A.; Barlaz, M. A.; Barofsky, D. F.; Field, J. A. Quantitative Determination of Fluorochemicals in Municipal Landfill Leachates. Chemosphere 2011, 82 (10), 1380-1386. https://doi.org/10.1016/j.chemosphere.2010.11.072.

(4) Allred, B. M.; Lang, J. R.; Barlaz, M. A.; Field, J. A. Orthogonal Zirconium Diol/C18 Liquid Chromatography-Tandem Mass Spectrometry Analysis of Poly and Perfluoroalkyl Substances in Landfill Leachate. J. Chromatogr. A 2014, 1359, 202-211. https://doi.org/10.1016/j.chroma.2014.07.056.

(5) Li, B.; Danon-Schaffer, M. N.; Li, L. Y.; Ikonomou, M. G.; Grace, J. R. Occurrence of PFCs and PBDEs in Landfill Leachates from Across Canada. Water. Air. Soil Pollut. 2012, 223 (6), 3365-3372. https://doi.org/10.1007/s11270-012-1115-7.

(6) González-Barreiro, C.; Martínez-Carballo, E.; Sitka, A.; Scharf, S.; Gans, O. Method Optimization for Determination of Selected Perfluorinated Alkylated Substances in Water Samples. Anal. Bioanal. Chem. 2006, 386 (7), 2123-2132. https://doi.org/10.1007/s00216-006-0902-7. 